\title{
Study of the current stressing in nanomanipulated three-dimensional carbon nanotube structures
}

\author{
D. C. Cox, R. D. Forrest, P. R. Smith, V. Stolojan, and S. R. P. Silva a) \\ Nano-Electronics Centre, Advanced Technology Institute, University of Surrey, Guildford, Surrey, GU2 7XH, \\ United Kingdom
}

(Received 2 December 2004; accepted 27 May 2005; published online 11 July 2005)

\begin{abstract}
We report the fabrication of free-standing carbon nanotube structures. The welding of individual carbon nanotubes to other nanotubes and metal substrates has been performed, on a selective basis, to produce joints of both good electrical conductivity and mechanical integrity, without the need for a joining material. As a result of this unique process, we study the damage to the microstructure of the nanotube as a function of current. When the current densities are in excess of $2 \times 10^{6} \mathrm{~A} / \mathrm{cm}^{2}$, particular care must be taken with regard to the quality of the nanotube and the heat dissipation. This is crucial for the use and application of nanotubes in any future device structure for it gives the upper limits to the "average" current density calculations. This process now allows for the fabrication of bespoke carbon nanotube devices for the prototyping of device performance. (C) 2005 American Institute of Physics. [DOI: 10.1063/1.1990268]
\end{abstract}

The electronic and microstructural properties of carbon nanotubes have been well documented, ${ }^{1}$ and many future technological applications have been proposed with them acting as key components. These applications range from reinforcing filler materials in composites, to extremely highperformance electronic components. However, to realize electronic applications, such as quantum wires, ${ }^{2}$ ballistic conductors, ${ }^{3}$ microchip interconnects, ${ }^{4}$ and transistors, ${ }^{5}$ the need to reproducibly fabricate connections between individual nanotubes and electrodes has been identified as a major impediment. ${ }^{6}$ Indeed, while theoretical studies, and limited experimentation, have demonstrated carbon nanotubes as showing exceptional promise, ${ }^{7}$ to date only the most rudimentary devices have been constructed. Most of these devices are based on small numbers of nanotubes, often limited to individual tubes, and have been constructed using suspensions of nanotubes deposited onto substrates, where electrical contacts have been deposited previously. ${ }^{8}$ Furthermore, for devices of any complexity, high repeatability and selectivity are essential. Methods that show promise with regard to manipulating and placing nanotubes at discrete locations are becoming available, and connecting and "soldering" of individual nanotubes with atomic force microscope ${ }^{9}$ and scanning electron microscope $e^{10,11}$ has been demonstrated. Scaling these techniques to produce higher throughput is problematic, however, as nanotube connections can take several minutes per joint, as well as problems associated with contaminants from the joining material.

In this letter we show a technique to select, manipulate, and produce nanotube joints with good mechanical and electrical integrity, without the need for a solder material, and with significantly reduced time taken per connected joint. The technique is based on controlled heating of the joints formed between nanotubes and substrates, and relies on careful control of applied voltages and subsequent currentinduced heating. We also demonstrate the negative effects of allowing excessive heating to take place in the nanotubes and its effect on the crystalline structure of the nanotube.

\footnotetext{
a) Author to whom correspondence should be addressed; electronic mail: s.silva@surrey.ac.uk
}

Arc-discharge-produced nanotubes of high crystalline quality were loaded in a scanning electron microscope (SEM) that includes electrical feedthroughs connected to a pair of sharp tungsten tips and the specimen substrates. The tips can be moved independently of the SEM stage and each other by three-axis piezo sliders, with a minimum step size of $20 \mathrm{~nm}$ and up to $5 \mathrm{~mm}$ total travel [Fig. 1(a)]. A LABVIEW program is used to run two Keithley 238 source/current meters connected to the tips and stage that provide a means of electrically characterizing, cutting, and welding individual nanotubes. In addition to the substrates containing the nanotubes further substrates are mounted on the stage onto which

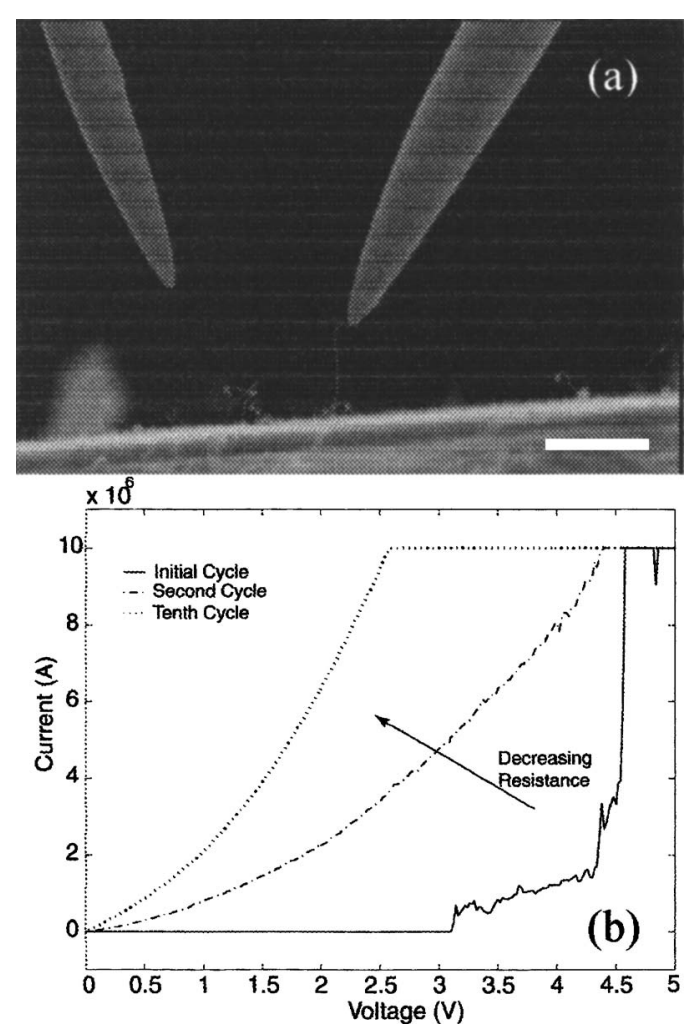

FIG. 1. Secondary electron micrograph of the tungsten tips and substrate in the SEM (scale bar is $10 \mu \mathrm{m})(\mathrm{a}), I$ - $V$ curves for the nanotube-tip-substrate connection (b). 


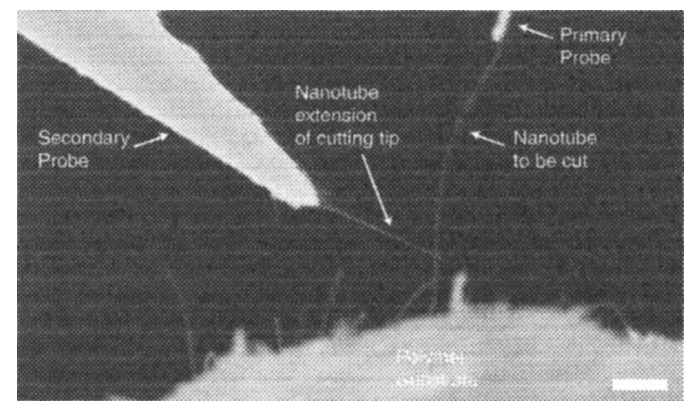

FIG. 2. Micrograph showing selected nanotube about to be cut. (Scale bar is $1 \mu \mathrm{m}$.

selected nanotubes can be deposited. These deposition substrates are typically metal wires and foils, and thin-film depositions on insulating substrates.

To select a nanotube for the manipulation process, one of the tips is brought close to a single nanotube on its substrate, and the gap is reduced until contact is made. Often, electrostatic attraction pulls the nanotube onto the end of the tip, relieving the need for accurate positioning. A voltage is then applied across the nanotube to establish an electrical contact. Initially, a poor contact is achieved and the measured current rarely exceeds a few nanoamperes, until a threshold voltage $(\sim 3 \mathrm{~V})$ is reached [Fig. 1(b)]. As the voltage is raised further, the current then rapidly increases by several orders of magnitude up to a predetermined limit of $1 \times 10^{-5} \mathrm{~A}$. This limit is imposed to prevent excessive current causing tube damage or failure. Subsequent cycling of the voltage over a typical range 0 to $5 \mathrm{~V}$ with a current limit still imposed results in a lowering of the resistance with each successive cycle down to approximately $150 \mathrm{k} \Omega$ for the connection shown. We have, however, seen values as low as $12 \mathrm{k} \Omega$ depending on the carbon nanotube and substrate. The tip-tonanotube contact is now mechanically robust and electrically active. After typically ten cycles, completed in under a minute, stable $I-V$ behavior is obtained, with no further conditioning. As can clearly be seen in Fig. 1(b), initially, the current limit of $1 \times 10^{-5} \mathrm{~A}$ is observed at around $4.5 \mathrm{~V}$, but following cycling, the same current can be achieved at lower than $2.5 \mathrm{~V}$. We believe this conditioning behavior is due to ohmic heating taking place at the connection, caused by the large current densities, facilitating alloying at the connection.

Cutting nanotubes to a predetermined length is by utilizing the second tip. A nanotube is connected between the first (primary) tip and the substrate, using the welding technique, and then a voltage of typically $1-2 \mathrm{~V}$ is applied. On bringing the second (cutting) tip, with a similar voltage, into contact with the nanotube, at the point cutting is required, and then raising the voltage on the cutting tip while limiting the current that can flow into the primary tip to zero, causes all the current to flow in the lower portion of the nanotube attached to the substrate. This results in a small region of around 100-150 $\mathrm{nm}$ vaporizing near to the point of contact of the cutting tip, leaving the desired length of nanotube attached to the first tip. Following the cutting procedure it becomes possible to move the nanotube attached to the tip to a new substrate and connect it as previously shown. It is also possible to connect additional nanotubes to the previously deposited ones. As with attaching to substrates, the nanotubes are brought into contact at the desired point on the previously deposited nanotube and cut off from the tip. However, when nanotubes are connected, no conditioning behavDownloaded 30 Mar 2009 to 131.227 .178 .132 . Redistribution subject

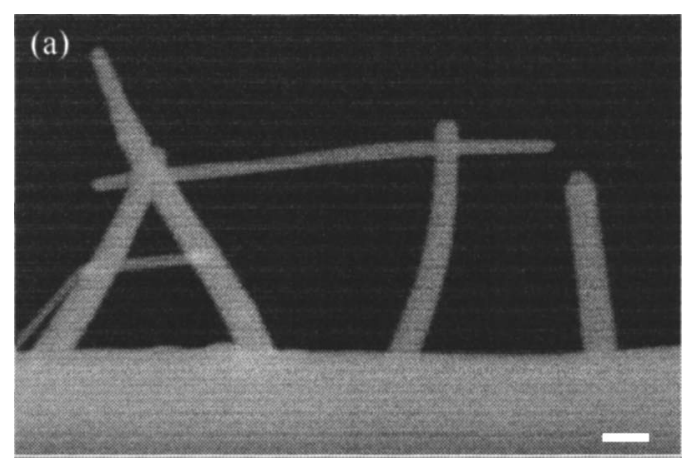

(b)

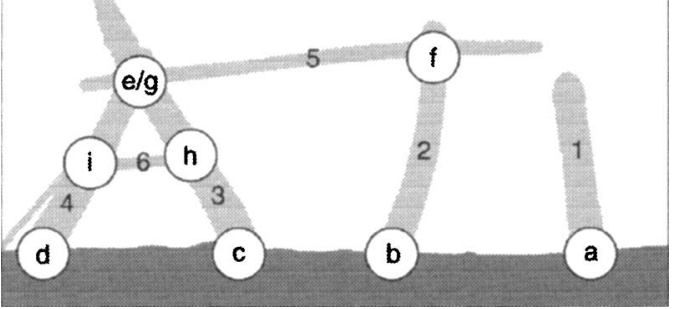

FIG. 3. Nanotube construction of letters A, T, and I using six nanotubes. Secondary electron micrograph of nanotube construction (scale bar is $300 \mathrm{~nm}$ ) (a), and its schematic diagram showing order in which the nanotubes were attached and the connections formed (b). The nanotubes were placed in the order shown numerically (1-6) and the connections made in the alphabetical order also shown in the schematic diagram.

ior is observed, and the $I-V$ behavior of the connection is instantly reproducible.

As a demonstration of the versatility of this new technique, Fig. 3 shows the letters A, T, and I constructed from carbon nanotubes and deposited onto the edge of a chromium foil. The height of the three letters is approximately $1.5 \mu \mathrm{m}$, occupying a length of less than $4 \mu \mathrm{m}$. The figure demonstrates the most important requirements necessary for the production of three-dimensional nanotube structures; the ability to pick and place a single nanotube onto a substrate with a specific length (letter "I"), the ability to attach a second nanotube to the first (letter "T"), and the ability to connect nanotubes together that have previously been attached to a chosen substrate (letter "A"). All of the connections are mechanically strong as well as electrically active.

Using the technique just described, we have probed the effect of current conduction on the microstructure of individual nanotubes as a function of current density. At the limits of current carrying capability, massive structural damage is done to the crystalline quality of the tube, where in the extreme, portions of tube are seen to vaporize. What is less clear is to what extent does the welding process possibly alter or damage the tubes microstructurally. To this end, we have carried out further experiments wherein portions of nanotube are exposed to high currents, and other portions exposed to lower, or no current. Figure 4(a) shows an example of how this is achieved, where it can be seen that a single nanotube is isolated by a tip, and connected some distance from the free end. Now, by carefully limiting the maximum current flow, we can expose the connected side of the tube to current stressing, while the free end sees no flow of current. Following current stressing, we place the whole tip complete with nanotube into the transmission electron microscope (TEM). Figure 4(b) shows the unstressed side of the tube with Figures 4(c) and 4(d) showing the stressed sides of a nanotube that was limited to $80 \mu \mathrm{A}$ maximum to AIP license or copyright; see http://apl.aip.org/apl/copyright.jsp 


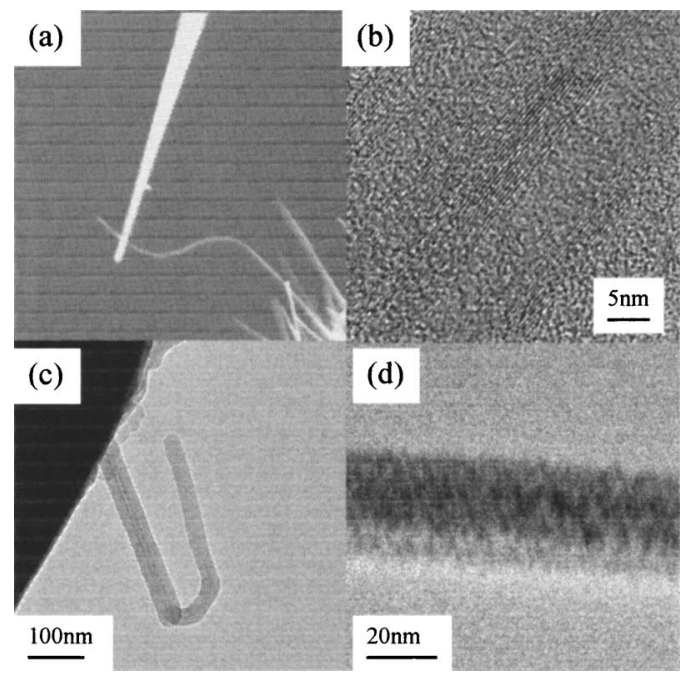

FIG. 4. Micrographs showing SEM image of connected nanotube (a), TEM of unstressed side (b), low-magnification TEM of current stressed side (c), and high-magnification of current stressed side (d).

current in the connected side. The micrograph of the unstressed side of the nanotube [Fig. 4(b)] shows well-defined concentric layers consistent with a multiwalled carbon nanotube. By comparison the micrograph of the stressed side [Figs. 4(c) and 4(d)] shows no well-defined structure, although the overall dimensions of the tube appear unchanged.

Electron energy loss spectra of the stressed tubes also reveal the change in their structure. For both the stressed and unstressed tubes, the concentration of $s p^{2}$-type bonded carbon atoms is measured using the normalized area of the $\pi^{*}$ peak $^{12}$ and is found to $100 \% s p^{2}$, suggesting that stressing the tube has not resulted in the formation of $s p^{3}$-type bonds. The sharp peak, at $\sim 294 \mathrm{eV}$, in the unstressed tube carbon $K$-edge, characteristic of crystalline graphitic carbon, disappears completely in the $K$-edge of the stressed tube, confirming the amorphisation of the tube following the flow of a high current close to $80 \mu \mathrm{A}$ through the tube $(\sim 70$ $\left.\times 10^{6} \mathrm{~A} / \mathrm{cm}^{2}\right)$. Tubes have been welded with maximum currents of $2 \mu \mathrm{A},\left(\sim 2 \times 10^{6} \mathrm{~A} / \mathrm{cm}^{2}\right)$ and subsequent TEM examination of the tube structure near to the contacts shows no perceptible structural damage.

The average failure power for the tubes measured in this study is $\sim 80 \mu \mathrm{W}$, which is considerably smaller than the failure power levels in other tubes of comparable sizes and type $^{13}(>1 \mathrm{~mW})$. In multiwalled nanotubes a number of conduction mechanisms have been proposed, with each giving rise to different failure thresholds. Nanotubes exhibiting ballistic conduction have failure powers in excess of $5 \mathrm{~mW},{ }^{14}$ although ballistic nanotubes in free-standing geometry show slightly lower power levels $(2.5 \mathrm{~mW}) .{ }^{15}$ The failure of nanotubes exhibiting diffusive conduction ${ }^{16}$ showed a $12 \mu \mathrm{A}$ per layer saturation current and subsequent failure at $\sim 500 \mu \mathrm{W}$. Oxidation assisted by the presence of intrinsic defects of those arisen as a result of the high current density was cited as the failure mechanism. In addition, in both diffusive and ballistic conduction, the point of failure has been correlated to specific defects present within the structure ${ }^{15,17}$.

The arc deposited multiwalled nanotubes studied here appear to show a broad double peaked distribution in failure power, peaking at $\sim 180$ and $20 \mu \mathrm{W}$. There appears to be two key factors that control the power that can be sustained before failure in this free-standing geometry. We believe a contributing factor to the lower power failure is the additional stress loading on the tubes, which exacerbates any failure at defects possibly due to localized heating. Indeed, anecdotally, nanotubes with significant curvature forced upon them fail at lower power, which follows due to the free-standing structures having poorer heat dissipation. The radiative power loss from an ideal black body of the dimensions of a nanotube at $2000 \mathrm{~K}$ is at least an order of magnitude less than the failure power threshold reported here. This limits the cooling to conduction through the body of the tube to the contacts. The cross-sectional area at the contacts, through which all the heat must flow, can be extremely small, and high temperatures can be reached at relatively low power levels, which in turn will increase resistivity in localized sections of the tube and cause failure at these defects. The lower peak in the distribution may be a result of specific defects of mechanical stress/strain in the tube.

In conclusion, we demonstrate that it is possible to weld nanotubes to metal contacts and to each other with both good electrical and mechanical quality. The procedure is fast enough to produce relatively complex structures and uses only the number of nanotubes required. We show that in electrical conduction through the tubes, heat dissipation is crucial if high current density devices are to be fabricated. Any structural or mechanical defect compromises the integrity of the tube, particularly at current densities in excess of $2 \times 10^{6} \mathrm{~A} / \mathrm{cm}^{2}$. This procedure may be utilized as a technique to construct carbon nanotube-based devices and connections in electronic applications of higher complexity than previously available by other techniques.

The authors acknowledge the support received from the EPSRC, UK, in the form of a Portfolio Partnership grant.

${ }^{1}$ Carbon Nanotubes: Synthesis, Structure, Properties and Application, edited by M. S. Dresselhaus, G. Dresselhaus, and P. Avouris (Springer, New York, 2000).

${ }^{2}$ S. J. Tans, M. H. Devoret, H. J. Dai, A. Thess, R. E. Smalley, L. J.

Geerligs, and C. Dekker, Nature (London) 386, 474 (1997).

${ }^{3}$ C. T. White and T. N. Todorov, Nature (London) 393, 240 (1998).

${ }^{4}$ J. Li, Q. Ye, A. Cassell, H. T. Ng, R. Stevens, J. Han, and M. Meyyappan, Appl. Phys. Lett. 82, 2491 (2003).

${ }^{5}$ R. Martel, Nat. Mater. 1, 203 (2003).

${ }^{6}$ R. H. Baughman, A. A. Zakhidov, and W. A. de Heer, Science 297, 787 (2002).

${ }^{7}$ P. L. Mc Euen, M. S. Fuhrer, and P. Hongkun, IEEE Trans. Nanotechnol. 1, 78 (2002).

${ }^{8}$ J. Appenzeller, J. Knoch, R. Martel, V. Derycke, S. J. Wind, and Ph. Avouris, IEEE Trans. Nanotechnol. 1, 184 (2002).

${ }^{9}$ W. Henk, Ch. Postma, M. de Jonge, Z. Yao, and C. Dekker, Phys. Rev. B 62, 10653 (2000).

${ }^{10}$ F. Banhart, Nano Lett. 1, 329 (2001).

${ }^{11}$ Madsen D. Nørgard, K. Mølhave, R. Mateiu, A. M. Rasmussen, M. Brorson, C. J. H. Jacobsen, and P. Bøggild, Nano Lett. 3, 47 (2003).

${ }^{12}$ S. D. Berger, D. R. McKenzie, and P. J. Martin, Philos. Mag. Lett. 57, 285 (1988).

${ }^{13}$ W. Y. Jang, N. N. Kulkarni, C. K. Shih, and Z. Yao, Appl. Phys. Lett. 84, 1177 (2004).

${ }^{14}$ Y. X. Liang, Q. H. Li, and T. H. Wang, Appl. Phys. Lett. 84, 3379 (2004).

${ }^{15}$ P. G. Collins, M. Hersam, M. Arnold, R. Martel, and Ph. Avouris, Phys. Rev. Lett. 86, 3128 (2002).

${ }^{16} \mathrm{Ph}$. Poncharal, C. Berger, Y. Yi, Z. L. Wang, and W. A. de Heer, J. Phys. Chem. B 106, 12104 (2002).

${ }^{17}$ P. J. de Pablo, S. Howell, S. Crittenden, B. Walsh, E. Graugnard, and R. Reifenberger, Appl. Phys. Lett. 75, 3941 (1999). 